\title{
A VUELTAS CON EL GÉNERO: CRÍTICAS Y DEBATES ACTUALES EN LA HISTORIOGRAFÍA FEMINISTA
}

\author{
GRAPPLING WITH GENDER: CRITICAL VIEWS \\ AND CURRENT DEBATES IN FEMINIST HISTORIOGRAPHY \\ Inmaculada Blasco Herranz* \\ Universidad de La Laguna, España
}

\begin{abstract}
RESUMEN: El objetivo de este artículo es invitar a la reflexión y provocar la discusión en torno al uso del género para el análisis del pasado. Con este objetivo en mente, se desgranan diferentes críticas que se han vertido sobre el género y su uso: desde la creciente insatisfacción de la propia Joan W. Scott, referente para las historiadoras e historiadores en la conceptualización del género como categoría de análisis, pasando por la a veces más radical propuesta de las historiadoras poscoloniales, hasta los recientes cuestionamientos que apuntan tanto al binarismo implícito en el concepto como a los efectos reificadores de su uso. A través de la exposición de estas tres líneas de crítica, se plantea la reapertura de los debates teórico-epistemológicos que subyacen a las mismas. Por una parte, el carácter y manejo de las categorías analíticas en historia, y, por otro lado, la articulación histórica de las diferencias y de las relaciones de poder, así como la implicación de ambos en la construcción de los sujetos históricos.
\end{abstract}

PALABRAS CLAVE: género, Joan W. Scott, historia postcolonial, binarismo de opuestos, relaciones de poder, diferencia.

ABSTRACT: The purpose of this article is to invite reflection and inspire discussion on the use of gender in historical analysis. To this aim, the paper looks at three lines of criticism to gender and its use: from the dissatisfaction of Joan W. Scott, a leading advocate on gender as a category of historical analysis; through the sometimes more radical proposal by postcolonial historians; to the recent questioning of the oppositional binary implicit in the concept as well as the reifying effect of its use. Through the discussion of these criticisms, I suggest the benefits of re-opening epistemological and theoretical debates, on the one hand, of the nature and operation of analytical categories in history and, on the other hand, on historical articulation of difference and power relations, together with their part in the construction of historical subjects.

KEYWORDS: gender, Joan W. Scott, postcolonial history, oppositional binary, power relations, difference.

* Correspondencia a: Inmaculada Blasco Herranz. Departamento de Geografía e Historia e Instituto Universitario de Estudios de las Mujeres (ULL). Facultad de Humanidades. Sección de Geografía e Historia. Apartado 456 (38200 San Cristóbal de La Laguna-Santa Cruz de Tenerife) - iblasco@ull.edu.es - https//orcid.org/0000-0003-2870-6876

Cómo citar: Blasco Herranz, Inmaculada (2020). "A vueltas con el género: críticas y debates actuales en la historiografía feminista»; Historia Contemporánea, 62, 297-322. (https://doi.org/10.1387/hc.20000).

Recibido: 22 junio, 2018; aceptado: 5 febrero, 2019.

ISSN 1130-2402 - eISSN 2340-0277 / (C) 2020 UPV/EHU 


\section{Introducción}

El objetivo central de este artículo es invitar a la reflexión y promover un diálogo en torno al género como categoría analítica para aproximarnos a la indagación del pasado. Mi punto de partida es que resulta preciso, quizás ya desde hace un tiempo, detenerse a repensar y reevaluar nuestra comprensión y manejo del género como historiadoras e historiadores. En España (que es el contexto que conozco mejor, si bien no creo que las peculiaridades se impongan sobre lo compartido) también «pusimos a funcionar» la categoría en nuestras investigaciones, lo cual se ha traducido en una rica y prolija producción histórica desde finales del siglo XX. No obstante, el uso del género se ha convertido en una operación en ocasiones mecánica, que da por sentada la plena validez de la categoría analítica en sí. Quizás por este motivo, entre otros, las críticas recientes que se han vertido sobre el género como concepto de análisis histórico han pasado casi desapercibidas. Si bien recogimos las tempranas dudas formuladas por la propia Scott en 1999, ${ }^{1}$ ni la investigación empírica - con fuerte carga de crítica teórica - de las que podemos denominar «historiadoras poscoloniales», ni dos artículos recientes que han sistematizado esta crítica, el de Jean Boydston (2008) y el de Anna Krylova (2016), han constituido objeto de discusión en la historiografía española. ${ }^{2}$

Mi propuesta concreta consiste en reconstruir los términos del debate desarrollado en las tres últimas décadas, acerca de la categoría, presentando algunas de las críticas que se han formulado, tanto hacia su uso como en torno a sus significados. Un primer apartado lo dedico a exponer los motivos de insatisfacción de Joan W. Scott con el género, desde finales de los años noventa hasta la actualidad. En segundo lugar, introduzco las principales críticas que algunos trabajos de «historia poscolonial» vertieron, ya desde los años noventa, sobre la capacidad del género para explicar otros pasados no occidentales ni modernos. Por último, expongo y evalúo las debilidades que Jeanne Boydston y Anna Krylova encuentran en el género tal y como ellas entienden que ha acabado dotándose

1 ARESTI, 2006, p. 231.

2 Véase el reciente debate publicado en la revista Ayer con el título «Historia de las mujeres y de género: pasado y futuro». VV.AA., 2016. Hemos optado por atender a las aportaciones a este debate elaboradas desde la historia, siendo conscientes de la existencia de otras aproximaciones críticas al género desde el ámbito, por el ejemplo, de la filosofía y de la epistemología. Un ejemplo de ello es FRAISSE, 2016 [2014]. 
de significado y arrastrando un modelo analítico concreto. Así mismo reflexiono sobre las propuestas de resignificación sugeridas por estas autoras, que movilizan también una modificación del aparato teórico explicativo tanto de las configuraciones del poder como de la construcción de la diferencia y de los sujetos históricos.

A través de este análisis, mi objetivo último no es tanto cuestionar el género sino más bien posibilitar una reapertura del debate teórico a partir de un uso problematizado del mismo que nos permita seguir pensando la articulación histórica de la diferencia y del poder, dos cuestiones que estaban en la base de las búsquedas iniciales de la historiografía feminista y, en particular, de quienes apostaron por el género. Por lo tanto, esta aproximación crítica a los recientes cuestionamientos del género para el análisis histórico, además de proponerse explorar las discusiones historiográficas que estos han suscitado, está animada por el afán de conectar con debates teóricos más amplios que han venido dinamizando la(s) teorización(es) feminista(s) en las últimas décadas.

\section{Joan Scott encuentra problemas en el género}

La principal crítica al género formulada tanto por Jeanne Boydston como por Anna Krylova ha sido que el binarismo de opuestos (oppositional binary) ha quedado incrustado en el concepto, tanto en el proceso de su teorización como en el uso que se ha hecho del mismo en la investigación empírica. ${ }^{3}$ El rechazo al empleo del género como un conjunto de categorías fijas (hombre y mujer), y opuestas, ya lo había expresado Joan W. Scott, como señalan estas autoras, desde la edición revisada de Gender and the Polititcs of History (1999). En efecto, en el prefacio a esa edición de 1999 (escrito, por lo tanto, más de diez años después de la primera edición), Scott afirmaba que, aunque el género parecía haber sido una categoría de análisis útil en los ochenta, porque «tenía un efecto extraño, desestabilizador», en los noventa había perdido «su capacidad para

${ }^{3}$ BOYDSTON, 2008; y KRYLOVA, 2016. Resulta tentador contraargumentar que, desde nuestra comprensión del género, a día de hoy, quizás menos atrapada por el binarismo, siempre hemos pensado el género en su variabilidad, pero respondería a una percepción desde un presente que solo muy recientemente ha comenzado a integrar una concepción de género más flexible. 
sorprendernos y provocarnos». ${ }^{4}$ Que estuviera perdiendo su «filo crítico» Scott lo ligaba al hecho de que en su uso ordinario había pasado a ser sinónimo de mujeres y de sexo o diferencias de sexos, de reglas sociales sobre los sexos. Para Scott, por lo tanto, el problema con el género radicaba en que las investigaciones históricas que lo usaban, por un lado, asumían la distinción sexo/género, y, por otra parte, aunque reconocían las diferencias culturales y nacionales, las presentaban como variaciones de un tema universal en las que «género siempre significa lo mismo, esto es, una relación asimétrica, acaso antagonista, entre las mujeres y los hombres, la cual establece las funciones de cada uno dentro de unos espacios y unas actividades por separado». Por este motivo, concluía, se encontraba usándolo cada vez menos y, en su lugar, prefería utilizar «diferencias entre los sexos y acerca del sexo como un concepto variable desde un punto de vista histórico»..$^{5}$ Ya entonces pedía, en consonancia con esta valoración, nuevos conceptos - una renovación del vocabulario analítico- para conservar el filo crítico que ella asociaba a la investigación feminista. ${ }^{6}$

Se puede inferir que el problema que Scott detectaba entonces con respecto al uso del género en los estudios históricos era, fundamentalmente, el del esencialismo, tanto del concepto mujeres como del de género, que se concretaba en la invariabilidad histórica de sus significados, a pesar incluso de la continua insistencia en su mutabilidad. Por estos motivos, le parecía necesario historizar la categoría «mujeres» (guiada por el trabajo de Denise Riley), y analizar sexo, género y diferencia sexual en tanto que efectos y no como puntos de partida del análisis. Una prevención, recomendaba Scott, para acercarse al estudio del pasado pertrechados de este concepto consistiría en «descartar la idea (inherente a la noción de género como categoría) de que hay algo fijo o que conozcamos de antemano en cuanto a los términos 'hombres' y 'mujeres' y a la relación entre ellos».

${ }^{4}$ SCOTT, 2008a [1999], p. 14.

5 SCOTT, 2008a [1999], p. 15. La anterior cita, en p. 247.

6 SCOTT, 2008a [1999], p. 15. El último libro de Scott, si bien no incorpora «gender» en el título, sí que lo hace a lo largo del análisis, cuyo objetivo es «to open - not to definitively close - a conversation about the place of gender equality in the discourse of secularism». SCOTT, 2018, p. 7.

7 SCOTT, 2008a [1999], p. 253. El trabajo de Denise Riley, Am I that Name? Feminism and the Category of Women in History, fue uno de los referentes más importantes en la obra de Scott que incidió en su concepción del género y también de la historia del feminismo. 
Que el sexo era el dato fundacional del género, que el género se empleaba como sinónimo de mujeres, que la definición de mujeres - aunque no significaba lo mismo en diferentes momentos históricos - se sustentaba sobre un elemento común universal para todo tiempo y espacio, eran los problemas más extendidos que Scott detectaba en el uso que las historiadoras habían hecho del género durante los años noventa del siglo pasado. Para contrarrestar el esencialismo y la invariabilidad, su propuesta concreta era entender el género como una forma de conocimiento que organiza nuestra percepción del mundo, de la naturaleza y del sexo. Esta comprensión desplazaría nuestra indagación hacia la articulación e implementación del conocimiento sobre la diferencia entre sexos. Sin duda, la reflexión y discusión-desmontaje de la dicotomía sexo/género en el ámbito de la teoría feminista postestructuralista (desde Butler hasta Donna Haraway) que tuvo lugar en los años noventa impregnó la redacción de este prefacio. ${ }^{8}$

Casi diez años más tarde, en 2008, a propósito de un foro de discusión organizado por la American Historical Review, precisamente con el objetivo de hacer un balance de la historiografía de género en la última década, Scott colaboró con una breve intervención titulada «Unanswered Questions», en la que volvía a insistir en casi lo mismo que en 1999.9 Añadía, como novedad, la desvinculación del carácter de categoría analítica que tanto impulso había contribuido a dar en la academia su artículo. Insistía Scott en que lejos de constituir un «tratado programático o metodológico», el género era útil solo si lo entendíamos de manera flexible: «Cuando el género es una pregunta abierta sobre cómo estos significados se establecen, qué significan y en qué contextos emergen, sigue siendo una categoría útil de análisis histórico». ${ }^{10}$ Dado que las autoras participantes en el foro de discusión abordaban el impacto del género en enclaves geográficos y tradiciones historiográficas muy diversas, Scott reconocía que «a partir de estos artículos, resulta evidente que el género no es un

8 BUTLER, 2002 [1993]; y HARAWAY, 1995 [1991].

9 SCOTT, 2008b. Reunía artículos sobre el impacto que había producido la intervención teórica de Scott en torno al género en diferentes historiografías. El de Dyan Elliott sobre la historia medieval, el de Gail Hershatter y Wang Zheng sobre China, el de Maria Bucur en torno a Europa oriental, el de Heidi Tinsman sobre América Latina, y el de Joanne Meyerowitz, acerca de la historia de los Estados Unidos.

10 SCOTT, 2008b, p. 1429. Lo del «tratado», en p. 1423. Se han traducido las citas solo en caso de no existir traducción al castellano de la obra que las contiene. 
concepto con parámetros o referentes fijos universalmente aplicable». ${ }^{11}$ La tarea había de ser entonces investigar, a través de los materiales históricos que examinamos, cómo se producen y transforman históricamente los significados sobre los cuerpos sexuados.

Como en 1999, estimaba necesario, por una parte, historizar la categoría «mujeres» (tener presente su carácter inestable e inconstante). ¿Acaso se puede equiparar, se preguntaba, la entidad colectiva que designamos como «mujeres» con los rasgos físicos compartidos de las mujeres? Sería el siglo XIX, como concluía Denise Riley, el que con su atención a la naturaleza y al cuerpo llevaría a enfatizar la sexualidad de las mujeres y también a fijar la oposición hombre/mujer como la verdad sobre la diferencia sexual. Por otro lado, sería preciso deconstruir la oposición sexo/género, es decir, la idea de que el género se construye sobre el sexo biológico y que este último es la cimentación invariable sobre la que se sustenta el género. Por último, insistía en que habría de interesarnos no solo (o no tanto) cómo el género construye la política (una línea de análisis cultivada con cierta profusión a partir de su noción de género como «forma primaria de significar el poder») sino también cómo la política conforma el género. En consecuencia, cómo se modelaron los significados cambiantes de la diferencia sexual y cómo se articularon con otros conceptos en apariencia alejados del sexo.

Dos años más tarde, en 2010, volvía a publicar una reflexión en torno al género, esta vez en la revista Diogenes, que consistía en un intento de rememorar, ya en un nuevo contexto de teorizaciones feministas y sobre sexualidad, el sentido de sus propias búsquedas con el género. Retomaba la idea del cuestionamiento del mismo como categoría de análisis, y subrayaba el objetivo que había guiado, en 1986, su esfuerzo teorizador, que no había sido otro que «historizar todos los términos», o, dicho de otro modo, mostrar cómo las palabras significan diferentes cosas en distintos contextos. De ahí que Foucault, esgrimía, le hubiera resultado útil para investigar cómo se estableció cierto conocimiento en torno a la diferencia sexual como natural, y cómo y cuándo un régimen de verdad fue reemplazado por otro, es decir, para explorar las diferentes formas específicas de organización de la diferencia sexual. Su indagación tenía como objetivo, reconocía, desestabilizar «el poderoso influjo de la biología al abrir todo aspecto de la identidad sexuada al cuestionamiento, incluyendo la pre-

${ }^{11}$ SCOTT, 2008b, p. 1492. 
gunta de si hombre/mujer, o masculino/femenino era el contraste que se invocaba». ${ }^{12}$

Sin embargo, era precisamente su objetivo de historización lo que no se estaba llevando a cabo con el uso del género, de ahí que en su valoración final concluía que este concepto había dejado de ser útil y eficaz. Redundaba en la demanda, por un lado, de atender a los significados de hombres y mujeres (no importa tanto la palabra sino lo que significa en distintos contextos), en lugar de manejarlos como definiciones fijas; y, por otro lado, de preguntarse cómo llegaba a construirse la diferencia sexual en lugar de, simplemente, describir los roles asignados. En resumen, reiteraba la necesidad de desnaturalizar la categoría «mujeres» y deconstruir el binario sexo/género (género es útil si lo entendemos como clave para desentrañar «sexo»). Esta vez parecía dejar más claro que la propuesta incluía también la desnaturalización del género (entendida como una relación hombre/mujer jerárquica e invariable, casi siempre en oposición heteronormativa).

En 2011, Scott presentaba una nueva definición de género, derivada de lo que parecía una modificación de sus marcos teóricos de referencia, entre los que acogía de manera central y explícita al psicoanálisis lacaniano. Así, género ya no era tan solo «una construcción social, una forma de organizar las divisiones sociales, económicas y políticas del trabajo según las normas de la diferenciación sexual. Es, más bien, una tentativa, cultural e históricamente específica, de resolver el dilema de la diferencia sexual, de asignar un significado fijo a lo que en última instancia no puede fijarse». ${ }^{13}$ Algunas voces han interpretado esto como el punto de llegada (no definitivo, tratándose de Scott, y más aún si leemos su último libro de 2018) de un camino, iniciado en 1999, a la búsqueda de la resolución de la tensión que había quedado impresa en el género entre la fijación de oposiciones y la desestabilización de la mismas. ${ }^{14}$

\section{El desafío al género desde la historia poscolonial}

Ya desde los años noventa, mientras aplicábamos el género confiadamente y Scott empezaba a mostrar su insatisfacción con la forma en

\footnotetext{
12 SCOTT, 2011a, p. 100.

13 SCOTT, 2011b, pp. 4-5.

14 KRYLOVA, 2016, p. 311.
} 
la que se estaba usando, hubo trabajos empíricos que lo cuestionaban de manera más contundente que la propia Scott. Particularmente reveladoras de los límites del género han sido las investigaciones de historiadoras «postcoloniales». Los trabajos de Ôyèronké Oyèwúmí (1997), Tani E. Barlow (2004) y Afsaneh Najmabadi (2005), entre otras, han contribuido a mostrar las insuficiencias del género para el análisis histórico de procesos e identidades fuera de Occidente. Entre ellos, quien hizo una crítica más temprana, profunda y radical al género fue la primera, con su The Invention of Women. ${ }^{15}$ Resulta interesante, y merecedor de reflexión, que solo veinte años más tarde haya sido traducido al castellano por el grupo de feministas decoloniales que lo presenta como «obligada lectura para los feminismos descoloniales de la Abya Yala», en la medida en que «nos invita a sospechar de las verdades que hemos heredado de la teorización feminista blanca y que hemos contribuido a universalizar al aplicarlas a nuestros contextos». ${ }^{16}$

Aunque puedan verterse críticas sustanciales sobre su trabajo, la propuesta de Oyèwúmí nos lleva por un camino de cuestionamientos y de interrogaciones, en ocasiones de reediciones de viejas preguntas, muy necesarias para la buena salud de la historiografía feminista, incluso cuando fuera elaborada a mediados de los años noventa y la tengamos que situar y entender en ese contexto. Oyèwúmí investiga el impacto que tuvo la colonización británica sobre la concepción (que no visión, como se explicará más adelante) del mundo de un pueblo yoruba precolonial del suroeste de Nigeria. Entre otras cosas, concluye que el proceso de colonización introdujo el género y las «mujeres» como categoría de clasificación e identificación social, una conceptualización que también estructura los estudios académicos sobre los yoruba.

15 OYÈWÚMÍ, 2017 [1997]. De las otras dos autoras, BARLOW, 2004, y NAJMABADI, 2005. Se entiende aquí poscoloniales en el sentido de que elaboran una crítica al empleo de categorías de la modernidad occidental para analizar contextos ni occidentales ni modernos.

${ }^{16}$ ESPINOSA, 2017, p. 14. Traducido por el Glefas (Grupo Latinoamericano de Estudio, Formación y Acción Feminista). http://glefas.org/la-invencion-de-las-mujeresoyeronke-oyewumi/. Las feministas decoloniales cuestionan radicalmente la mirada del feminismo occidental («eurocentrado») tanto por lo que respecta a los modelos de interpretación de las formas de organización social (sexual, racial, colonial), como a la verdad dominante sobre la dominación de las mujeres. El programa Contratiempo dedicó uno de sus espacios a la presentación de la traducción (http://www.contratiempohistoria.org/?p=6500). Véase también OYÈWÚMÍ, 2002. 
La tesis analítica de Oyèwúmí es que en la categoría género está implícita la narrativa «occidental-moderna» que, por una parte, arrastra valoraciones de las culturas europeas y de la historia occidental y, por otro lado, no puede dar cuenta de realidades en las que esto no funcionó así. Los problemas que detecta en la categoría género y que Scott había señalado en $2008^{17}$, aunque no parece dialogar con ella, derivan de la universalización de presupuestos no generalizables al objeto de investigación del que se ocupa. Es decir, que una noción particular de género se ha aplicado a la comprensión de otros contextos geográficos e históricos en los que pudieron existir otras configuraciones de la diferencia. A partir de ahí, Oyèwúmí deconstruye los supuestos que, desde su perspectiva, subyacían al concepto «género» tal y como se estaba empleando en los noventa (si bien su apreciación sobre el género puede resultar bastante generalista y, desde el presente, parecernos que ya no lo estamos usando como un concepto tan inmutable y fijo como Oyèwúmí lo interpreta), y muestra cómo su objeto de estudio no se ajusta a ninguno de ellos.

En primer lugar, ante el presupuesto de que el género es un principio central de organización social, sostiene que no todas las sociedades están organizadas sobre la base de género, ya que en el caso del pueblo yoruba precolonial serían más bien el estatus y la edad (seniority) los marcadores de diferencia/identidad significativos y potenciales criterios de jerarquización social. ${ }^{18}$ En consecuencia, y a diferencia de lo que trajo el colonialismo, la biología no se tradujo en categorías sociales jerarquizadas. En segundo lugar, para Oyèwúmí, el género alude siempre a relaciones de poder que se traducen en jerarquías (de manera que, a veces, cuando hablamos de género siempre presuponemos una relación de subordinación de las mujeres con respecto a los hombres). Esto, afirma la autora, sucedió precisamente cuando el estado colonial patriarcal impuso sobre los pueblos yoruba la categoría «mujeres» como identificable, definida por su anatomía y subordinada a los hombres en toda situación. Las mujeres quedaron excluidas entonces de las estructuras del estado, en contraste con su situación en la organización socio-política yoruba precolonial, en la que poder no estaba determinado por el género.

17 Ver nota 11.

18 La crítica de Oyèwúmí suscita un cuestionamiento más amplio hacia la universalización de categorías analíticas elaboradas en el contexto de la modernidad Occidental. Véase CHAKRABARTY, 2008 [2000]. 
En tercer lugar, el género suele presuponer la existencia previa de un cuerpo sexuado hombre/mujer (una crítica que ya estaban haciendo Joan Scott y Judith Butler, pero desde otras fuentes teóricas). Este presupuesto, que Oyèwúmí denomina «determinismo corporal», derivaría de la «centralidad somática de muchos años en pensamiento occidental». El determinismo corporal o la «ideología del determinismo biológico» consiste en la «idea de que la biología provee la base para la organización del mundo social» o, dicho de otro modo, que ciertos órganos determinan la posición social y por lo tanto el cuerpo es la base de los roles que llevan a la exclusión/inclusión. ${ }^{19}$ Según Oyèwúmí, en los pueblos yoruba que estudia, el cuerpo no era un fundamento de roles sociales ni de identidad ni jerarquías. La única distinción existente, que no constituye una diferencia que se traduzca en jerarquía social, fue la de la reproducción. La explicación a esta somatocentricidad occidental, a esta omnipresencia del cuerpo en las sociedades modernas occidentales remite a que se percibe por la vista, por eso importa (y el lenguaje expresa esa relevancia) la «visión» del mundo y no el «sentido» del mundo, algo que a su vez se insertaría en una dualidad cuerpo/mente. En cierta discrepancia con Thomas Laqueur, que sitúa en el tránsito de la premodernidad a la modernidad un punto de inflexión en la concepción del sexo único a los dos sexos, Oyèwúmí sostiene que en la tradición occidental, desde la Antigüedad, se impone una percepción de dos cuerpos, dos sexos, dos categorías que derivan de lo que se ve. ${ }^{20}$ Afirma la autora que, aunque Laqueur tenga razón en los cambios que se produjeron en el tránsito a la modernidad, no obstante, y su propio trabajo intenta demostrarlo, existe un sustrato común en el mundo Occidental desde la Antigüedad: la centralidad del cuerpo en la construcción de categorías sociales.

Por último, el género presupone siempre un binario (hombre y mujer) en relación de dominación, lo que descarta la posibilidad de imaginar en el pasado (y también el presente y en el futuro) otras distinciones sobre cuerpos sexuados que no se hagan sobre la diferencia binaria y que no funcionen como vehículos de dominación. Al análisis de una determinada sociedad con esta categoría que, según Oyèwúmí, asume estos presupuestos, lo llama etnocentrismo de género. Como otros etnocentrismos, opera a través de la universalización de nociones aparentemente neutrales, cuyo

19 OYÈWÚMÍ, 2017, p. 15.
20 LAQUEUR, 1994. 
sesgo queda oculto, y tiene gran impacto sobre la sociedad analizada en varios sentidos. Para empezar, crea somatocentricidad e inscribe el género en la sociedad que analiza. En segundo lugar, inyecta problemas - y debates - que no existen en otras sociedades. En este sentido, se considera que el feminismo, «a pesar de su actitud radical, exhibe las mismas características etnocéntricas e imperialistas de los discursos occidentales que busca subvertir». Dicho de otro modo, las feministas no escaparían a la lógica occidental, al desplegar como universales conceptos como patriarcado y mujeres que a su vez derivarían de otros como biología, anatomía, cuerpo y vista. Frente a esto, defiende que «el marco de referencia de una cultura debe identificarse y describirse en sus propios términos antes de hacer cualquier tipo de afirmaciones gratuitas como las que se hacen sobre el patriarcado y otras desgracias sociales». ${ }^{21}$

Entre las críticas que ha recibido La Invención de la mujer destacamos la más inmediata de Bibi Bakare Yusuf, que consideró problemático el «esencialismo» (en este caso cultural) y el «autenticismo» que deriva de su apelación al conocimiento local, así como los supuestos teóricos en torno al lenguaje y al discurso sobre los que se fundamenta. ${ }^{22} \mathrm{Si}$ bien el nativismo planea sobre esta investigación, su acercamiento al género resulta enormemente sugerente para nuestro trabajo de análisis de la conformación y cambio de las diferencias sexuales en el pasado por diferentes motivos. Muestra no solo la posibilidad de que hayan existido otras formas de articular diferencias sociales, y por lo tanto de configuración de los sujetos y su agency. También subraya la particularidad de nuestro régimen de verdad, y de las categorías de análisis que generamos en su seno (en particular, desde el ámbito de las ciencias sociales). Dicho de otro modo, si emprendemos nuestras indagaciones pertrechados con género (o una determinada conceptualización del mismo, a veces implícita), no solo encontraremos género, sino que también lo consolidaremos como criterio de clasificación y organi-

21 OYÈWÚMÍ, 2017, p. 68. La cita anterior, en p. 55.

22 BAKARE-YUSUF, 2011. Una crítica parecida, que califica el trabajo de nativista, en SEGATO, 2003. A mi juicio, sin embargo, la veta más nativista de Oyèwúmí emerge claramente en su último trabajo de 2016, What Gender Is Motherhood? Changing Yorùbá Ideals of Power, Procreation, and Identity in the Age of Modernity, que entronca con la reflexión epistemológica de los estudios decoloniales latinoamericanos. Una visión crítica muy clarificadora de las posiciones decoloniales desde una visión poscolonial, que expone las diferencias entre historia decolonial y poscolonial, en SABSAY, 2013. 
zación social. Para la autora, esta dificultad para deshacerse de la visión prevaleciente de que las jerarquías sociales se construyen a partir de la diferencia anatómica es precisamente la razón por la cual no hemos llevado a sus últimas consecuencias la afirmación de que el género es una construcción histórica que se articula en contextos culturales específicos y cambia históricamente. Si el género es una construcción social, habría que reconocer que «hubo entonces un tiempo específico (en diferentes sitios arquitectónico/culturales) en el cual "fue creado" y, en consecuencia, un tiempo previo en el que la creación no había ocurrido (...). Entonces, resulta lógico suponer que en algunas sociedades la construcción de género jamás llegó a suceder». ${ }^{23}$

En síntesis, asumir el análisis de Oyèwúmí supone aceptar que seguimos pensando a las mujeres como grupo homogéneo determinado biológicamente, constitutivamente victimizado y desprovisto de poder; y al género como una relación de poder basada en el sexo que siempre ha existido aunque se va transformando históricamente (algo muy parecido, sino igual, al patriarcado). De hecho, se podría afirmar que así como género se convirtió en sinónimo de mujeres en una gran parte de nuestros trabajos históricos, relaciones de género ha llegado a equiparase a patriarcado. Esto no deja de tener sentido, si atendemos al objetivo inicial de la historiografía feminista, pero sería interesante seguir reflexionando al respecto: ¿Por qué dejamos de utilizarlo? ¿Por qué lo volvemos a utilizar o empleamos género en su lugar? ${ }^{24}$ ¿Qué vino a cambiar género en el análisis de las relaciones de poder, subordinación o exclusión de las mujeres con respecto a «patriarcado»?

Otro estudio similar en su aproximación al género y en algunas de sus conclusiones es el de Afsaneh Najmabadi sobre el Irán Qajar. Al igual que Oyèwúmí, esta autora muestra que el género fue una construcción occidental que conformó el proceso de modernización de Irán. De su análisis sobre las figuras homoeróticas entre finales del siglo XVIII y comienzos del siglo XX, establece unas conclusiones muy similares a las de Oyèwúmí: que pensar el género como hombre/mujer es un imperativo «muy moderno»; que haber arrastrado esta matriz definitoria compuesta de dos términos ha tenido como consecuencias su naturalización

23 OYÈWÚMÍ, 2017, p. 51.

${ }^{24}$ Significativo de la reconsideración de su uso para el análisis histórico de la Edad Moderna es el foro en Gender \& History el año pasado. WIESNER-HANKS, 2018. 
y su «atemporalización»; que las narrativas resultantes han contribuido a «renaturalizar el sexo»y a «binarizar el género». ${ }^{25}$

\section{Críticas recientes: binarismo y reificación}

A estas investigaciones que analizan cómo occidente ha impuesto categorías de clasificación e identificación en el proceso histórico colonial, la historiadora norteamericana Jeanne Boydston añade otros ámbitos de estudio histórico que, en ocasiones muy tempranamente, también han cuestionado el binarismo implícito en el género. ${ }^{26}$ Centrada en la historia de los Estados Unidos, la autora presentaba, en su artículo de gran impacto publicado en 2008, trabajos muy tempranos sobre la comunidad afro-americana que, siguiendo la crítica del feminismo negro, concluían que ni las distinciones entre hombres y mujeres ni la feminización implicaban necesariamente subordinación. En segundo lugar, destacaba aquellas investigaciones sobre nativos americanos (en general más recientes), que incidían en la idea de que el género no tenía por qué ser binario, ni primario como marcador de identidad, ni ligado a categorías corporales fundamentadas en la biología, ni a un concepto de poder basado en el dominio o el control. En tercer lugar, introducía aquellas indagaciones en historia «temprana» americana (siglos XVI y XVII) que han cuestionado que el género fuera siempre estructurante o vertebrador en las vidas de las mujeres coloniales (en estos contextos, el status es el que aparece como discurso de poder en el siglo XVII), y que han visibilizado las «transgresiones» del binario de opuestos. ${ }^{27}$ En este último caso, advierte Boydston, al ser tratadas como excepciones más que como alternativas, han seguido percibiéndose como «proto-binarias» o precuelas de la «ideología» de las esferas separadas. En opinión de esta autora, sin embargo, remitirían a una manera diferente de estructurar la relación entre hombre y mujer (más fluida, $y$ en la que el género no funcionaba de manera tan central como organizador del poder y de la autoridad).

25 NAJMABADI, 2005 y 2006.

26 BOYDSTON, 2008.

27 «Oppositional binary» es la expresión que emplean tanto Boydston como Krylova. Se ha optado por traducirlo como «binarismo de contrarios» o «binario de opuestos». Una variante del debate se ha iniciado en el terreno de los estudios feministas sobre conflictos. Véase SAEIDI, 2012, 818. 
Hasta aquí, las críticas de Boydston al género resultan coherentes con lo planteado por las historiadoras poscoloniales antes citadas, a cuyos trabajos añade investigaciones sobre tradiciones culturales no blancas y periodos históricos no modernos. Más problemático me parece el último ámbito de contextos históricos seleccionado por Boydston en su ejemplificación de que el género, entendido como un binario, no siempre tiene encaje o no siempre resulta útil para captar esos pasados. En este caso, el género (que remite siempre a un «binario de opuestos») se considera inadecuado para explorar la variabilidad y transformaciones de los discursos de género en Europa y EEUU en el tránsito del siglo XIX al XX. La problemática que se suscita es diferente, pues se trata no tanto de hacer visibles configuraciones de diferencia (sexual) no binarias o no articuladas por relaciones de poder sino más bien de cómo entendemos y analizamos las alternativas no binarias en un contexto en el que parece que lo binario era el sentido común o la verdad sobre la diferencia sexual. Boydston sugiere que también en este caso las historiadoras han generado una narrativa del triunfo del «binario» que no solo no da cuenta de una realidad que escapa al mismo sino que ha subordinado, marginalizado o considerado excepcionales las alternativas al binarismo. Su conclusión de que el binario pudo no haber tenido una existencia tan generalizada y dominante como se ha supuesto nos llevaría a entender la historia del género a finales del siglo XIX y comienzos del XX (en América del Norte) «como un discurso mucho más complejo de lo que la categoría binaria reconoce». ${ }^{28} \mathrm{Y}$ aporta en su apoyo algunos ejemplos de estudios sobre dicho periodo que muestran la ausencia tanto de una heteronormatividad clara como de ese binario de opuestos, que le llevan incluso a afirmar que el binario hombre/mujer fue un epifenómeno.

Se podría objetar que aquí Boydston está defendiendo algo diferente e incluso contradictorio con lo que las «historiadoras poscoloniales» argumentan. Para estas, el género (binarismo de opuestos) es una construcción moderna occidental (para algunas, cuyo despliegue completo se produce en la modernidad liberal - también para Laqueur- y para otras -Oyèwúmí - que deriva de una vieja tradición de pensamiento occidental desde la Antigüedad) que se ha pretendido universalizar (o sea, presentar como universal sin mostrar su parcialidad histórica y cultural). Para Boydston, el binarismo (casi siempre se añade «oppositional», porque se

${ }^{28}$ BOYDSTON, 2008, p. 569; la anterior cita, p. 568. 
sobreentiende que hemos asumido con «género» una relación siempre binaria y siempre en oposición jerárquica) tampoco puede servirnos para analizar la modernidad occidental, en un momento, el fin de siglo, que podría considerarse como de eclosión de la misma (con el cientifismo, el nacionalismo y el imperialismo como telones de fondo). Y no nos serviría porque la realidad nos muestra tantas excepciones al mismo, que no hacen sino poner en cuestión si no su existencia sí su poder regulador, su extensión y su hegemonía. ${ }^{29}$ Parece que la autora quiere romper con una visión teleológica y lineal articulada en torno al ideal decimonónico de domesticidad que sería el paroxismo del binarismo: ni lo de antes fue proto-binario ni lo binario fue en realidad tan dominante y prevaleciente. Entonces, ¿se podría afirmar que fue en algún momento hegemónico el binarismo y la domesticidad que se generó en torno a aquel?

El problema de fondo de la propuesta de Boydston es que, al querer desprenderse de lo binario no deja de tenerlo en cuenta para buscar «lo que no fue binario» y/o lo que no estuvo permeado por relaciones de poder, y presentarlo como mucho más habitual o al menos como no marginal. La nueva dicotomía que podría llegar a estar implícita en esta búsqueda es la de lo no binario frente a lo binario. Es decir, la búsqueda de lo no binario forma parte de la respuesta o rechazo al imperativo de lo binario impreso en el concepto género hasta hace poco.

Anna Krylova se une a Jeanne Boydston (y a otras que recoge esta última) con su artículo de hace un par de años, «Gender Binary and the Limits of Poststructuralist Method». ${ }^{30}$ Como Boydston, no desea abandonar la categoría (ya que reconoce que se trata de un «logro de varias generaciones»), pero afirma dar un paso más, pues considera necesario desarrollar una alternativa al aparato teórico que subyace al género, a la que Boydston solo apunta someramente. Esta búsqueda parte de la necesidad de dar cuenta de fenómenos de heterosexualidad fuera de lo normativo,

${ }^{29}$ Se podría argumentar que estas reflexiones han sido posibles porque las lentes con las que miramos el pasado se han visto influidas, desde los años noventa, por el resquebrajamiento, precisamente, del dominio del binarismo, al abrirse la posibilidad de que existan más de dos géneros o no géneros y por la visibilidad y creciente legitimidad social de las sexualidades no heteronormativas.

${ }^{30}$ KRYLOVA, 2016. Como novedad con respecto a (y actualización de) las críticas que se han efectuado sobre el género y sus efectos en las narraciones históricas, añade (aunque el funcionamiento excluyente es el mismo que en el caso de otros sujetos) el ocultamiento de las experiencias que no encajan en el esquema binario debido al «heterosexismo» de la historia de las mujeres y del género (gays-lesbianas, queer, transgénero y third gender...). 
como el que analiza en su investigación sobre las mujeres soldado en el ejército soviético durante la II Guerra Mundial. ${ }^{31}$

Krylova se detiene más que Boydston en la reflexión sobre el efecto reificador del género, es decir, el efecto que produce su uso en la práctica, de reproducir aquello que se quiere cuestionar, algo que ya percibió Scott en su intento de, en palabras de Krylova, «resistir, sin éxito, (...) a sus propiedades binarias». A sus ojos, este quedar atrapada por la categoría resulta de sus consustanciales efectos reificadores derivados del enfoque postestructuralista, lo que ejemplificaría a la perfección el análisis de Scott en Only Paradoxes to Offer ${ }^{32}$ Entonces, el problema radicaría no tanto en los usos de otras, como Scott señalaba, sino en cómo la propia Scott lo ha concebido y aplicado en sus trabajos empíricos. El «método postestructuralista» sería el responsable último de la atribución de los significados con los que hemos connotado al género (oposición, diferenciación exclusiva y exhaustiva, jerarquía y dominación). Y esto nos habría llevado a considerar como excepciones, subversiones o transgresiones aquello que debería ser visto como alternativas. En concreto, Krylova localiza las causas de las dificultades analíticas que ha suscitado el género, en su versión postestructuralista, en las operaciones de diferencia basadas en Derrida y en el concepto de poder foucaultiano (si bien Boydston ya había mostrado su insatisfacción con el uso de uno y otro para el análisis de la diferencia y del poder, respectivamente). Dicho de otro modo, el «binarismo de opuestos» sería un producto de las bases postestructuralistas (foucaultiana del poder y derrideana de la diferencia), que impregnan la teorización que Scott hizo del género en los años ochenta (sistema coherente - fijo pero inestable en su constitución-, que se automodifica y se autoperpetúa; y siempre ligado a desigualdad de poder). Este tipo de análisis, si seguimos la argumentación de Krylova, produciría la paradoja de posibilitar la crítica a los sistemas binarios fijos, pues al deconstruirlos se muestra su inestabilidad, al mismo tiempo que los refuerza, cancelando la posibilidad de alternativa, desde el momento en que se parte de la premisa de que todo se desarrolla dentro del sistema binario. ${ }^{33}$

31 KRYLOVA, 2010.

32 KRYLOVA, 2016, p. 309.

33 Incluso, añade Krylova, se obvian materiales que no encajan en las expectativas normativas. Una crítica similar desarrolla, en el mismo número de la revista Gender \& History, Mary Louise Roberts, quien, a través de la problematización del concepto de crisis de género, diagnostica que las historiadoras quedan atrapadas en esa misma lógica binaria que buscan deconstruir y criticar. 
Scott reproduciría esto mismo, aunque no fuera su objetivo, en sus trabajos empíricos, particularmente en Only paradoxes to offer ${ }^{34} \mathrm{Al}$ analizar la construcción del binarismo de género, acabaría, según Krylova, reificando esta misma representación binaria de la diferencia sexual. Este esquema le habría impedido imaginarse a Madeleine Pelletier pensándose como mujer de otra manera que no fuera dentro del binario, de ahí que la interprete como una negación del ser mujer; o le habría llevado a calificar de transgresión la ocupación que hace Olympe de Gouges del espacio público-político (cuando, para Krylova, es muy probable que dicha ocupación obedeciera a una lógica de género no binaria).

Por último, a Krylova tampoco le parece adecuada la salida que propone Scott a esta paradoja, es decir, recurrir al psicoanálisis lacaniano para indagar la formación de la identidad normativa. ${ }^{35}$ El desplazamiento del análisis a cómo se resuelven los discursos normativos en el plano psíquico no terminaría de romper con el binarismo implícito en el género ni con la imposibilidad de generar alternativas desde los sistemas de dominación heteronormativa (que se quedarían en meras transgresiones, rupturas y fantasías).

La propuesta concreta de Krylova para examinar su objeto de investigación sería, en consecuencia, dejar de interpretar las identidades heterosexuales que no encajan en un esquema binario (sobreentiende, dos opuestos y uno subordinado al otro) como transgresiones individuales de una lógica omnipresente sino más bien como articulaciones identitarias que son posibilitadas por otras lógicas no binarias. De ahí que proponga una reconceptualización del género cuyo núcleo es la diferenciación entre dicotómico y binario, pues el primero no acarrearía necesariamente connotaciones positivas y negativas, y permitiría hablar de géneros opuestos (y cita a Marylin Frye) como «interdependientes, sin tener que ser [necesaria y transhistóricamente] dominante o subordinado (...)». ${ }^{36}$

34 SCOTT, 2012 [1996]. De este libro afirma Krylova que «lanza una crítica mordaz a los persistentes binarios de género que subyacen a los discursos históricos del feminismo pero también ofrece un estudio de caso del trabajo reificador de la representación binaria de la diferencia sexual en la literatura académica contemporánea». KRYLOVA, 2016, p. 314.

35 SCOTT, $2011 \mathrm{~b}$.

${ }^{36}$ KRYLOVA, 2016, p. 319. Aunque les resulta endeble la alternativa ofrecida por Krylova, Nerea Aresti y Miren Llona consideran apropiada la crítica al binarismo que formula esta autora. Por el contrario, desestiman su cuestionamiento de la ligazón permanente entre poder y género. Véase ARESTI y LLONA, 2019, p. 377. 


\section{Debates: poder y diferencia en la historia feminista}

Más allá de que podamos estar de acuerdo con parte de las críticas al género, como la propia Scott también lo ha expresado, estos cuestionamientos y las alternativas propuestas son susceptibles de ser discutidos, además de resultar bienvenidos para mantener vivo el debate teórico en el terreno de la historiografía feminista, cuya tendencia al empiricismo forma parte de los hábitos de la disciplina en general. A lo que apunta Krylova es a dos cuestiones que requieren nuestra atención, reflexión y discusión, y que subyacen a la historia de género (y la de las mujeres) desde sus inicios: cómo analizamos las relaciones de poder en el pasado y cómo se construye la diferencia. Aunque con menor énfasis y profundidad, la crítica al género se ha centrado, además de en el binarismo de opuestos, y ligándolo al mismo, en la universalización de ese binario como relaciones de poder. Por una parte, las poscoloniales (y con anterioridad las afroamericanas) habían cuestionado la universalización de las relaciones de dominación de hombres sobre mujeres en todos los contextos, derivada del énfasis del feminismo blanco y occidental en la diferencia sexual biológica (según la lógica del pensamiento occidental), como parte del etnocentrismo europeo. Así, para Oyèwúmí, «en muchos escritos feministas, la presuposición y utilización universal de las categorías de patriarcado y «mujer» resulta etnocéntrica y manifiesta la hegemonía de Occidente sobre otras clasificaciones culturales». ${ }^{37}$

Por otro lado, tanto Boydston como Krylova rechazan la noción de poder foucaultiano que, afirman, ha quedado implícita en el género tal y como lo formuló y manejó Scott. Más aún, consideran que refuerza el marco binario hombre/mujer en relación de oposición, y desecha como «inocentes» distinciones masculino/femenino que no encajan en esa diferenciación de poder. ${ }^{38}$ Boydston considera que Scott parte de una concep-

37 OYÈWÚMÍ, 2017, p. 59. Desde el feminismo decolonial latinoamericano también se afirma que el patriarcado fue impuesto en el proceso de colonización europea en «Abya Yala», lo cual ha reabierto el debate en torno a la existencia o no del patriarcado antes de la colonización. ESPINOSA, 2017, pp. 13-14.

${ }^{38}$ La discusión presenta muchas similitudes, salvando las distancias de aproximación analítica, con el debate que se planteó a finales de los años ochenta, particularmente en la historiografía francesa, entre una visión de las relaciones entre hombres y mujeres en clave de dominación y otra más «irenista», que destacaba los poderes de las mujeres y las relaciones de colaboración entre hombres y mujeres en el pasado. La autora afirmaba que la dominación masculina era una expresión más de la desigualdad de las relaciones sociales (puesto 
ción de la naturaleza del poder (a partir del trabajo de Foucault) que a su vez es un producto histórico en sí (euronorteamericano y occidental, del siglo XX). Se trataría de una concepción precisa que entiende el poder, por una parte, como un proceso consistente en crear ventaja con el objetivo de ejercer dominación y, por otro lado, que opera a través de formas de autodisciplina interiorizadas occidentales y burguesas (de ahí que las llamadas a resistir o liberarse del mismo solo sirvan para reforzarlo). Por último, a Boydston le parece simplificador que la posibilidad de cambio exista solo dentro del sistema de dominación que a su vez constituye a los sujetos. ${ }^{39}$ En resumen, apuntan a la necesaria historización del concepto de poder y de su relación con la conformación de los sujetos históricos.

No dejan de tener razón Boydston y Krylova en su valoración de cómo la historia de género ha trabajado el análisis del poder. Como afirma Wendy Brown no solo para el ámbito historiográfico, se ha podido, en algunos casos, «reiterar una comprensión del poder solo como dominación», con lo cual ha terminado por verse al poder «solo privilegiando u oprimiendo». No obstante, siguiendo con Brown, «no estamos simplemente oprimidas sino producidas a través de estos discursos, una producción que es históricamente compleja, contingente (...)». ${ }^{40}$ Dicho de otra forma, tanto a Boydston como a Krylova (y a otras muchas) se les olvidan las capacidades generativas que diferentes modalidades de ejercicio del poder tienen sobre la formación de sujetos históricamente situados. Por lo que, a su vez, se podría cuestionar que la visión que estas autoras tienen de la comprensión foucaultiana del poder resulta simplificadora y unidireccional en su funcionamiento. ${ }^{41}$ Todo ello sin dejar de reconocer, con Brown, que las teorías del poder y la historia existentes adolecen de limitaciones para analizar la construcción de los sujetos. ${ }^{42}$

que las relaciones entre los sexos son relaciones sociales): «Esta forma e desigualdad está inclusa en el funcionamiento de muchas sociedades cualquiera que sea su grado de desarrollo (...). No es específica de las sociedades occidentales, y hacerla salir un poco por todas partes no equivale a dar pruebas de un etnocentrismo exagerado». FARGE, 1991, pp. 89-90.

39 BOYDSTON, 2008, p. 563.

40 BROWN, 2005, p. 123 (la anterior cita, en p. 131).

41 Una excelente aproximación a la conformación de un sujeto específico, histórico y culturalmente situado, desde la «paradoja» de la sujeción-subjetivación, en MAHMOOD, 2004.

${ }^{42}$ La propuesta de Brown es seguir reflexionando desde la teoría feminista y de la historia guiadas por el propósito de «how to come to terms with the problem of the powers involved in the construction of subjects». En BROWN, 2005, p. 131. 
Puede resultar útil situar esta discusión en las coordenadas de lo que Scott denominaba como una de las tensiones más productivas de la historiografía feminista (la que nos mueve entre la política y la historización). Con el género se pretendía seguir buscando explicaciones para la dominación de las mujeres por los hombres, una búsqueda que había estado predominantemente guiada por el concepto de patriarcado. Como decía Scott a finales de los años ochenta, «[n]ecesitamos teorías que por lo menos rompan el esquema conceptual de esas viejas tradiciones filosóficas occidentales que han construido sistemática y repetidamente el mundo de manera jerárquica, en términos de universales masculinos y de especificidades femeninas». Y reconocía que fue esta búsqueda (feminista) la que le llevó al posestructuralismo. Desde ahí, el patriarcado era susceptible de ser historizado y, además, quedaba resituado en el plano de los significados (epistemologías) a través de la redefinición de la noción de discurso (campos discursivos) en conflicto (matizaba que, «al menos, desde la Ilustración»). ${ }^{43}$ La búsqueda de Scott (y su recurso al posestructuralismo) podría leerse, en consecuencia, como un intento de compensar un exceso de protagonismo femenino (y, por extensión, de «agency» naturalizada) en los relatos de historia de las mujeres que parecía haber contribuido a un descuido del análisis de los mecanismos de subordinación y jerarquización. La propia fundamentación epistemológica y filosófica de su aproximación postestructuralista al pasado impedía volver a explicar las jerarquías a partir de unos intereses universales y absolutos. El reto estaba en situar históricamente los intereses e identidades de los actores históricos, no entenderlos como resultado de posiciones estructurales sino de significados que cambian históricamente. Dicho de otro modo, se trataba de historizar los marcos del poder, dilucidar en qué términos específicos en una determinada cultura/contexto histórico se formularon las exclusiones y las jerarquías y fueron así percibidas por los sujetos históricos. Género, y no patriarcado, permitía esta aproximación a las diferentes formas, históricamente variables, de conocer la diferencia sexual. ${ }^{44}$

En cuanto a la segunda cuestión, la de cómo se configuran las diferencias, la propuesta de Krylova nos deja con las ganas de una mayor profundización. En este caso parece volver a la descripción pormenorizada

43 SCOTT, 1992 [1988], p. 90; las anteriores citas, en p. 87.

${ }^{4}$ SCOTT, 2008a [1999], p. 20. Para un análisis pormenorizado acerca de cómo Scott contribuyó a reformular las nociones de discurso, experiencia e identidad, véase CABRERA, 2006. 
de una identidad concreta (que, en este caso, se afirma que es dicotómica pero no binaria), pero no resuelve la cuestión de cómo se configura el sistema de diferencias que la posibilita (aunque sabemos que es alternativo al normativo, no sabemos cómo interactúa con este último, por ejemplo). Entonces, por un lado, si no es necesario entender la operación por la que se ha generado una diferencia concreta como objeto significativo, lógicamente tampoco interesa indagar en los mecanismos por los cuales se construye la diferencia. Pero, en caso de que suscitara interés, Derrida no parece un buen compañero de viaje para la explicación de estas configuraciones de diferencia (y, por ende, de construcción del significado) pues su uso conduce a un afianzamiento del binarismo en la comprensión de la diferencia sexual. El problema de fondo radica en las dudas acerca de la universalización de la naturaleza relacional de la diferencia, es decir, de la idea de que se es algo porque no se es otra cosa, lo cual articula la diferenciación y la exclusión. La historización de las operaciones de diferenciación suscita una serie de cuestionamientos a cómo se ha entendido generalmente que se constituyen las diferencias y estas devienen jerarquías. Habría que preguntarse si no sería más bien cada contexto el que daría la clave de operaciones de diferenciación particulares que las fuentes nos revelarían. Si (dependiendo del contexto) un concepto unitario no siempre contiene material negado o reprimido; si las oposiciones que se presentan como fijas no siempre son interdependientes en un sentido jerárquico (en ese caso, ¿se podría afirmar que solo la tradición filosófica occidental se fundamenta en oposiciones jerárquicas y que solo en ella los términos dominantes derivan su significado de los dominados, que posibilitan la definición de los primeros?; si el significado no siempre se configura a través del contraste implícito o explícito - es decir, que la definición positiva no siempre descansa sobre la negación o represión de algo que es representado como antitético - . Si esto fuera así, tendríamos que aceptar que la producción de diferencias y, por ende, de significados, no se ajusta siempre a una diferenciación «binaria» y jerárquica. Sin embargo, ¿podríamos asegurar que un modelo de género no binario (o, como en el ejemplo estudiado por Krylova, dicotómico pero no binario) nunca se configura sobre operaciones de diferenciación binaria y jerárquica?

Por último, la propuesta de expandir el género resulta atractiva, sobre todo porque encaja bien en los desarrollos socio-culturales actuales y en las críticas a la universalización del binarismo (si bien algunas de dichas críticas desechan completamente la categoría, como hemos visto, por arrastrar un significado, del cual no podría desprenderse, que implica 
también un proceso de dominación). Expandir el género, aunque puede abrir terrenos de investigación inéditos y empujarnos a imaginar modalidades de articulación de las diferencias no binarias, creo que presenta dos problemas que habría que intentar si no resolver, al menos discutir. El primero de ellos es el de la apuesta por dejar de buscar lo binario para buscar lo no binario, como se ha objetado más arriba. El segundo es que, en el completo rechazo a la conceptualización del poder y la subjetivación de Foucault, contempla la realidad en términos de lo normativo/ alternativo separadamente, generando una nueva dicotomía. Lo alternativo aparece como fruto de otra matriz no normativa de comprender relaciones de género, más flexible y no binaria. Parece claro que debe explorarse la articulación de otras modalidades de poder y de sujeto, y de la relación entre ellos, en sociedades no occidentales ni contemporáneas. No obstante, si asumimos el argumento de las feministas poscoloniales, ¿cómo podemos entender y explicar imaginarios de género no binarios dentro de la modernidad occidental binaria, dicotómica y basada en la biología?

Para concluir, la cuestión no es tanto, como se ha podido sugerir en ocasiones, si dejamos de emplear la categoría o no (si es que esta decisión puede llevarse a la práctica sin más), como quizás tampoco lo sea intentar controlar el significado del término (precisar más su definición, etc.). ${ }^{45}$ Se trataría de ser más conscientes de las limitaciones que tanto en su teorización como en su uso han subrayado las críticas. Pero principalmente, de enfrentarnos a los problemas y debates que emergen a la superficie, al hilo de dichas críticas y de las alternativas propuestas, que podrían sintetizarse en estas tres cuestiones: la universalización de categorías analíticas y nuestra relación de continua negociación con ellas; la articulación histórica de las diferencias (sexuales y otras) y las teorías del poder en la historia, así como la íntima implicación, que ha de ser desentrañada en el análisis empírico, de ambas cuestiones en la construcción de los sujetos históricos.

45 Para Scott, esto sería una tarea contradictoria con su compromiso con la crítica, e inútil, pues no hay significados transparentes y estables: «Mi trabajo ha alcanzado, después de todo, cierta legitimidad en el mundo de la teoría feminista en el que viajo; es una legitimidad que quiero preservar en sus propios términos. Sin embargo, la teoría que representa respalda la idea de que ningún pensamiento es inmune a la crítica, de que perseguir el conocimiento es un proceso interminable y a veces discontinuo, de que el futuro no puede quedar atado al pasado». En SCOTT, 2011b, p. 146. 


\section{Bibliografía}

AMADIUME, Ifi, Male Daughters, Female Husbands. Gender and Sex in an African Society, The University of Chicago Press, Chicago, 1987.

ARESTI, Nerea, «La categoría de género en la obra de Joan Scott», en Cristina Borderías (ed.), Joan Scott y las políticas de la historia, Icaria, Barcelona, 2006, pp. 223-232.

ARESTI, Nerea y LLONA, Miren, «Mary Nash, tras las huellas del feminismo histórico», Teresa ORTEGA, Ana AGUADO y Elena HERNÁNDEZ SANCOICA (eds.), Mujeres, dones, Mulleres, Emakumeak. Estudios sobre la historia de las mujeres del género, Cátedra, 2019, pp. 359-378.

BAKARE-YUSUF, Bibi, «Los Yoruba no hacen género»: una revisión crítica de "La invención de la Mujer: Haciendo un Sentido Africano de los Discursos Occidentales de Género" de Ôyèronké Oyèwúmí», africaneando. Revista de actualidad y experiencias, 5-1, 2011, pp. 25-54 (http://www.oozebap.org/ africaneando/africaneando-05.pdf. Consultado el 7 de abril de 2018).

BARLOW, Tani E., The Question of Women in Chinese Feminism, Duke University Press, Durhan y Londres, 2004.

BOYDSTON, Jeanne, «Gender as a Question of Historical Analysis», Gender \& History, 20-3, 2008, pp. 558-583.

BROWN, Wendy, E D GE W O R K. Critical Essays on Knowledge and Politics, Princeton University Press, Durham y Londres, 2005.

BUTLER, Judith, Cuerpos que importan. Sobre los límites discursivos del «sexo», Paidós, Barcelona, Buenos Aires, México, 2002. [Bodies that That Matter: On the Discursive Limits of «Sex», Routledge, Nueva York, 1993].

CABRERA, Miguel Ángel, «Lenguaje, experiencia e identidad. La contribución de Joan Scott a la renovación teórica de los estudios históricos», en Cristina BORDERÍAS (ed.), Joan Scott y las políticas de la historia, Icaria, Barcelona, 2006, pp. 233-257.

CHAKRABARTY, Dipesh, Al margen de Europa: pensamiento poscolonial y diferencia histórica, Tusquets, Barcelona, 2008. [Provincializing Europe. Postcolonial Thought and historical difference, Princeton University Press, Princeton, 2000].

DIVASSÓN, Blanca, Mujer, género y trabajo en Canarias en época contemporánea, 1868-1914 [tesis doctoral inédita]. Universidad de La Laguna, 2015.

ESPINOSA, Yuderkis, «Presentación. La importancia de leer a Oyěwùmí en América Latina», en LA INVENCIÓN DE LAS MUJERES. Una perspectiva africana sobre los discursos occidentales del género, Editorial en la frontera, Bogotá, 2017, pp. 7-26.

FARGE, Arlette, «La historia de las mujeres: Cultura y poder de las mujeres: ensayo de historiografía», Historia Social, 9, 1991, pp. 79-102. 
FRAISSE, Geneviève, Los excesos del género. Concepto, imagen, desnudez, Cátedra/PUV, Madrid, 2016. [Les excès du genre. Concept, image, nudité, Editions Lignes, París, 2014].

HARAWAY, Dona. J., Ciencia, cyborgs y mujeres: la reivención de la naturaleza, Cátedra, Madrid, 1995. [Simians, Cyborgs, and Women: The Reinvention of Nature, Routledge, Nueva York, 1991].

KRYLOVA, Anna, «Gender Binary and the Limits of Poststructuralist Method», Gender \& History, 28-2, 2016, pp. 307-323.

KRYLOVA, Anna, Soviet Women in Combat. A History of Violence on the Eastern Front, Cambridge University Press, Nueva York, 2010.

LAQUEUR, Thomas, La construcción del sexo. Cuerpo y género desde los griegos hasta Freud, Cátedra, Madrid, 1994. [Making Sex: Body and Gender from the Greeks to Freud, Harvard University Press, Cambridge/London, 1990].

MAHMOOD, Saba, The Politics of Piety: The Islamic Revival and the Feminist Subject, Princeton University Press, Princeton, 2004.

NAJMABADI, Afsaneh, «Beyond the Americas: Are Gender and Sexuality Useful Categories of Historical Analysis?», Journal of Women's History, 18-1, 2006, pp. 11-21.

NAJMABADI, Afsaneh, Women with Mustaches and Men without Beards. Gender and Sexual Axieties of Iranian Modernity, University of California Press, Berkeley, Los Angeles y Londres, 2005.

OYÈWÚMÍ, Ôyèronké, «Conceptualising gender: The Eurocentric foundation of feminist concepts and the challenge of African epistemologies», JENdA: A Journal of Culture and African Women Studies, 2-1, 2002, pp. 1-6.

OYÈWÚMÍ, Ôyèronké, The Invention of Women: Making an African Sense of Western Gender Discourses, University of Minnesota Press, Minneapolis y Londres, 1997 (traducción al castellano: LA INVENCIÓN DE LAS MUJERES. Una perspectiva africana sobre los discursos occidentales del género, Editorial en la frontera, Bogotá, 2017. (Descargado el 8 de mayo de 2017 en http://glefas.org/la-invencion-de-las-mujeres-oyeronke-oyewumi/).

OYÈWÚMÍ, Ôyèronké, What Gender Is Motherhood? Changing Yorùbá Ideals of Power, Procreation, and Identity in the Age of Modernity, Palgrave McMillan, Basingstoke, 2016.

RILEY, Denise, Am I that Name? Feminism and the Category of Women in History, MacMillan, Houndmills, 1988.

ROBERTS, Mary Louise, «Beyond "Crisis" in Understanding Gender Transformation», Gender \& History, 28-2, 2016, pp. 358-366.

SABSAY, Leticia, «Dilemas queer contemporáneos: ciudadanías sexuales, orientalismo y subjetividades liberales. Un diálogo con Leticia Sabsay», Íconos. Revista de Ciencias Sociales, 47, 2013, pp. 103-118. 
SAEIDI, Shirin, «Reconsidering Categories of Analysis: Possibilities for Feminist Studies of Conflict», Gender \& History, 24-3, 2012, pp. 799-824.

SCOTT, Joan W., «Igualdad versus diferencia: los usos de la teoría posestructuralista», Debate feminista, 3-5, 1992, pp. 85-104. [«Deconstructing equalityversus-difference: Or the uses of poststructuralist theory for feminism», $\mathrm{Fe}$ minist Studies, 14-1, 1988, pp. 33-50].

SCOTT, Joan W., Las mujeres y los derechos del hombre. Feminismo y sufragio en Francia, 1789-1944, Siglo Veintiuno, Buenos Aires, 2012. [Only Paradoxes to Offer. French Feminists and the Rights of Man, Cambridge y Londres, Harvard University Press, 1996].

SCOTT, Joan W., Género e historia. Fondo de Cultura Económica, México, 2008a. [Gender and the Politics of History. Revised edition, Columbia University Press, Nueva York, 1999].

SCOTT, Joan W., «Unanswered Questions», The American Historical Review, 113-5, 2008b, pp. 1422-1429.

SCOTT, Joan W., «Género: ¿Todavía una categoría útil para el análisis?», $L a$ manzana de la Discordia, 6-1, 2011a, pp. 95-101. [«Gender: Still a Useful Category of Analysis?», Diogenes, 57-1, 2010, pp. 7-14].

SCOTT, Joan W., The Fantasy of Feminist History, Duke University Press, Durham y Londres, 2011b.

SCOTT, Joan W., Sex and Secularism, Princeton University Press, Princeton, 2018.

SEGATO, Rita, «Género, política e hibridismo en la transnacionalización de la cultura Yoruba», Estudos Afro-Asiáticos, 25-2, 2003, pp. 333-363.

VV.AA., «Debate Historia de las mujeres y de género: pasado y futuro», Ayer, 104, 2016, pp. 249-276.

WIESNER-HANKS, Merry, «Forum Introduction: Reconsidering Patriarchy in Early Modern Europe and the Middle East», Gender \& History, 30-2, 2018, pp. 320-330.

\section{Financiación}

Este trabajo se ha elaborado en el marco del proyecto de investigación «Ciudadanía social, estado del bienestar y género en España. Nuevas aproximaciones desde la historia (1880-1936)». Referencia: PGC2018097232-B-C221 (MCIU/AEI/FEDER, UE). 


\section{Reconocimientos}

Agradezco a Javier Díaz Freire y a Mercedes Arbaiza por su invitación al seminario «Nuevos enfoques de la historia contemporánea: ¿Qué historia hacer hoy?» (Bilbao, junio de 2017), y a todas las y los miembros del grupo de investigación «Experiencia moderna», por alimentar tan dinámico espacio de discusión sobre cuestiones epistemológicas y teóricas en historia. También a Miguel Ángel Cabrera por las pequeñas conversaciones que mantenemos, que siempre resultan tan estimulantes. Todas las intervenciones en el Seminario Santos Juliá, donde se discutió recientemente una versión de este texto, son bienvenidas y de gran utilidad en mi reflexión. Dedico este ensayo a Mary Nash, por abrir tantos caminos en nuestra historiografía y, sobre todo, por intentar mostrarnos que la senda del debate es la que lleva a un conocimiento más fructífero del pasado. Y a la memoria de Saba Mahmood, cuya obra ha convulsionado profundamente mi manera de ver las cosas.

\section{Datos de la autora}

Inmaculada Blasco Herranz (iblasco@ull.edu.es) es doctora por la Universidad de Zaragoza y profesora de historia contemporánea en la Universidad de La Laguna. Desde 2008 ha compatibilizado la crianza de su hija con la docencia y con una labor investigadora que ha tenido como objeto el análisis de la construcción de identidades políticas en el movimiento católico femenino durante las dos primeras décadas del siglo XX. También se ha interesado por la exploración de la interacción entre las construcciones de género y de nación española a lo largo del Novecientos y por los discursos de género que permearon el reformismo social en los orígenes del Estado del bienestar. Sobre estos temas ha publicado diversos capítulos en libros colectivos y artículos en revistas especializadas como Historia Social, Gender \& History, Ayer, Pasado y Memoria, Arenal, Historia y Política, entre otras. 